Bull. Korean Math. Soc. 47 (2010), No. 6, pp. 1171-1180

DOI 10.4134/BKMS.2010.47.6.1171

\title{
SOME NEW CHARACTERIZATIONS OF WEIGHTED BERGMAN SPACES
}

\author{
SONGXIAO Li
}

ABSTRACT. In this paper we obtain some new characterizations for weighted Bergman spaces in the unit ball of $\mathbb{C}^{n}$.

\section{Introduction}

Let $D$ be the unit disk in the complex plane and $B$ be the unit ball in the $n$-dimensional complex Euclidean space $\mathbb{C}^{n}$. Let $z=\left(z_{1}, \ldots, z_{n}\right)$ and $w=$ $\left(w_{1}, \ldots, w_{n}\right)$ be points in $\mathbb{C}^{n}$, we write

$$
\langle z, w\rangle=z_{1} \bar{w}_{1}+\cdots+z_{n} \bar{w}_{n},|z|=\sqrt{\left|z_{1}\right|^{2}+\cdots+\left|z_{n}\right|^{2}} .
$$

Thus $B=\left\{z \in \mathbb{C}^{n}:|z|<1\right\}$. Let $d v$ be volume measure on $B$, normalized so that $v(B)=1$. We denote by $H(B)$ the class of all holomorphic functions on $B$.

Suppose $0<p<\infty$ and $\alpha>-1$, recall that the weighted Bergman space $A_{\alpha}^{p}$ consists of those functions $f \in H(B)$ for which

$$
\|f\|_{A_{\alpha}^{p}}^{p}=\int_{B}|f(z)|^{p} d v_{\alpha}(z)<\infty,
$$

where $d v_{\alpha}(z)=\left(1-|z|^{2}\right)^{\alpha} d v(z)$. When $\alpha=0$, we get the classical Bergman spaces, which will be denoted by $A^{p}$. In the setting of the unit disk, we denote $d v_{\alpha}(z)$ by $d A_{\alpha}(z)$.

There are many papers considered the characterization of weighted Bergman spaces, see $[2,3,6,7,9,11]$. In [2], among other results we obtained the following characterization for weighted Bergman spaces.

Theorem A. Assume that $f \in H(B)$ and $\alpha>-1$. If $\beta$ and $\gamma$ are real parameters satisfying

$$
\beta+\gamma=\alpha+p-(n+1)
$$

and

$$
-1<\beta<p-(n+1),-1<\gamma<p-(n+1),
$$

Received March 20, 2009; Revised March 3, 2010.

2000 Mathematics Subject Classification. Primary 32A36; Secondary 30D55.

Key words and phrases. weighted Bergman space, derivative-free, unit ball. 
then $f \in A_{\alpha}^{p}$ if and only if

$$
\int_{B} \int_{B}\left(\frac{|f(z)-f(w)|}{|1-\langle z, w\rangle|}\right)^{p} d v_{\beta}(z) d v_{\gamma}(w)<\infty .
$$

It is very important to give more characterizations for a function space, which is very useful to study operator theory on function spaces. For example, an $f \in H(B)$ is said to belong to the $\alpha$-Bloch space, denoted by $\mathcal{B}^{\alpha}(B)$, if

$$
\sup _{z \in B}\left(1-|z|^{2}\right)^{\alpha}|\nabla f(z)|<\infty .
$$

However it is difficult to study composition operators on $\alpha$-Bloch space by the last formula.

In [10], Zhang and $\mathrm{Xu}$ introduced a new metric by modifying the Bergman metric, i.e., they gave the following metric (see [10] for more details)

$$
F_{z}^{\alpha}(w)=\sqrt[2]{\frac{n+1}{2}} \frac{\sqrt[2]{\lambda_{\alpha}(|z|)|w|^{2}+\left(1-\lambda_{\alpha}(|z|)\right)|\langle w, z\rangle|^{2} /|z|^{2}}}{\left(1-|z|^{2}\right)^{\alpha}} .
$$

Using this new metric, they proved that $f \in \mathcal{B}^{\alpha}(B)$ if and only if

$$
\sup _{z, w \in \mathbb{C}^{n} \backslash\{0\}} \frac{|\nabla f(z) w|}{F_{z}^{\alpha}(w)}<\infty .
$$

Using (4), the boundedness and compactness of composition operators on $\alpha$ Bloch spaces have been completely characterized.

In this paper, we add some other characterizations, including derivativefree characterizations and mixture of derivative characterizations for weighted Bergman spaces in the unit ball of $\mathbb{C}^{n}$.

Throughout this paper, constants are denoted by $C$, they are positive and may differ from one occurrence to the other. The notation $a \asymp b$ means that there is a positive constant $C$ such that $b / C \leq a \leq C b$.

\section{Preliminaries and auxiliary results}

Let $\operatorname{Aut}(B)$ be the group of all biholomorphic maps $B$ into $B$. It is well known that $\operatorname{Aut}(B)$ is generated by the unitary operators on $\mathbb{C}^{n}$ and the involutions $\varphi_{a}$ of the form

$$
\varphi_{a}(z)=\frac{a-P_{a} z-s_{a} Q_{a} z}{1-\langle z, a\rangle}
$$

where $s_{a}=\left(1-|a|^{2}\right)^{1 / 2}, P_{a}$ is the orthogonal projection into the space spanned by $a$, i.e.,

$$
P_{a} z=\frac{\langle z, a\rangle a}{|a|^{2}},|a|^{2}=\langle a, a\rangle, P_{0} z=0
$$


and $Q_{a}=I-P_{a}$ (see, e.g. [11]). Moreover, $\varphi_{a}$ has the following well-known properties:

$$
\begin{gathered}
\varphi_{a}(0)=a, \varphi_{a}(a)=0,1-\left|\varphi_{a}(z)\right|^{2}=\frac{\left(1-|a|^{2}\right)\left(1-|z|^{2}\right)}{|1-\langle z, a\rangle|^{2}}, \\
1-\left\langle\varphi_{w}(z), w\right\rangle=\frac{1-|w|^{2}}{1-\langle z, w\rangle} .
\end{gathered}
$$

For $f \in H(B), z \in B$, let

$$
\nabla f(z)=\left(\frac{\partial f}{\partial z_{1}}(z), \ldots, \frac{\partial f}{\partial z_{n}}(z)\right)
$$

denote the complex gradient of $f$ and let $\widetilde{\nabla} f$ denote the invariant gradient of $B$, i.e.,

$$
(\widetilde{\nabla} f)(z)=\nabla\left(f \circ \varphi_{z}\right)(0) .
$$

Let $d \lambda(z)=\left(1-|z|^{2}\right)^{-n-1} d v(z)$. Then $d \lambda(z)$ is a Möbius invariant measure, that is, for any $\psi \in \operatorname{Aut}(B)$ and $f \in L^{1}(B)$,

$$
\int_{B} f(z) d \lambda(z)=\int_{B} f \circ \psi(z) d \lambda(z) .
$$

To prove the main results of this paper, we need some lemmas. We begin with the following result (see [11]).

Lemma 1. Assume that $f \in H(B), p>0$ and $\alpha>-1$. Then $f \in A_{\alpha}^{p}$ if and only if $|\widetilde{\nabla} f(z)|$ belongs to $L^{p}\left(B, d v_{\alpha}\right)$ and if and only if

$$
\int_{B}\left(1-|z|^{2}\right)^{p}|\nabla f(z)|^{p} d v_{\alpha}<\infty .
$$

Lemma 2 ([7]). Suppose $p>0, \alpha>-1,0 \leq q<p+2$ and $f \in H(B)$. Then $f \in A_{\alpha}^{p}$ if and only if

$$
\int_{B}|f(z)|^{p-q}|\widetilde{\nabla} f(z)|^{q} d v_{\alpha}(z)<\infty .
$$

Furthermore, the quantities

$$
\int_{B}\left(1-|z|^{2}\right)^{p}|\nabla f(z)|^{p} d v_{\alpha} \text { and }|f(0)|+\int_{B}|f(z)|^{p-q}|\widetilde{\nabla} f(z)|^{q} d v_{\alpha}(z)
$$

are comparable for $f \in H(B)$.

The following result is well known (see Proposition 1.4.10 [8] or Theorem 1.12 of $[11])$.

Lemma 3. Suppose $\alpha>-1$ and $t>0$. Then there exists a constant $C>0$ such that

for all $z \in B$.

$$
\int \frac{\left(1-|w|^{2}\right)^{\alpha} d v(w)}{|1-\langle z, w\rangle|^{n+1+\alpha+t}} \leq \frac{C}{\left(1-|z|^{2}\right)^{t}}
$$




\section{Main results and proofs}

Now we are in a position to state and prove the main results in this paper.

Theorem 1. Assume that $f \in H(B), p>0$ and $\alpha>-1$. If $\beta$ and $\gamma$ are real parameters satisfying (1) and (2), then $f \in A_{\alpha}^{p}$ if and only if

$$
\int_{B} \int_{B}\left(\frac{|f(z)-f(w)|}{\left|w-P_{w} z-s_{w} Q_{w} z\right|}\right)^{p} d v_{\beta}(z) d v_{\gamma}(w)<\infty .
$$

Proof. Suppose that (9) holds. Since

$$
\begin{aligned}
& \int_{B} \int_{B}\left(\frac{|f(z)-f(w)|}{|1-\langle z, w\rangle|}\right)^{p} d v_{\beta}(z) d v_{\gamma}(w) \\
\leq & \int_{B} \int_{B}\left(\frac{|f(z)-f(w)|}{\left|w-P_{w} z-s_{w} Q_{w} z\right|}\right)^{p} d v_{\beta}(z) d v_{\gamma}(w),
\end{aligned}
$$

from Theorem A we see that $f \in A_{\alpha}^{p}$.

Conversely, suppose that $f \in A_{\alpha}^{p}$. Let

$$
\begin{aligned}
I & =\int_{B} d v_{\beta}(z) \int_{B}\left(\frac{|f(w)-f(z)|}{\left|w-P_{w} z-s_{w} Q_{w} z\right|}\right)^{p} d v_{\gamma}(w) \\
& =\int_{B} d v_{\beta}(z) \int_{B} \frac{|f(w)-f(z)|^{p}}{\left|\varphi_{z}(w)\right|^{p}} \frac{d v_{\gamma}(w)}{|1-\langle z, w\rangle|^{p}} .
\end{aligned}
$$

Making the change of variables $w \mapsto \varphi_{z}(w)$ we have

$$
I=\int_{B} d v_{\alpha}(z) \int_{B} \frac{\left|f \circ \varphi_{z}(w)-f \circ \varphi_{z}(0)\right|^{p}}{|w|^{p}} \frac{d v_{\gamma}(w)}{|1-\langle z, w\rangle|^{2(n+1+\gamma)-p}} .
$$

From the proof of Proposition 10 of [2], we obtain

$$
I \leq C \int_{B} \int_{B} \frac{|f(z)-f(w)|^{p}}{|1-\langle z, w\rangle|^{p}} d v_{\beta}(z) d v_{\gamma}(w)<\infty .
$$

The proof of the theorem is complete.

Remark 1. Results of this type can be found in [5], where it was shown that an $f \in H(B)$ belongs to the Besov space $B_{p}(p>2 n)$ if and only if

$$
\int_{B} \int_{B}\left(\frac{|f(z)-f(w)|}{\left|w-P_{w} z-s_{w} Q_{w} z\right|}\left(1-|z|^{2}\right)^{\frac{1}{2}}\left(1-|w|^{2}\right)^{\frac{1}{2}}\right)^{p} d \lambda(z) d \lambda(w)<\infty .
$$

In the setting of the unit disk, $\left|w-P_{w} z-s_{w} Q_{w} z\right|$ is just $|z-w|$. From Theorem 1, we get the following corollary.

Corollary 1. Assume that $f$ is an analytic function in $D, p>0$ and $\alpha>-1$. If $\beta$ and $\gamma$ are real parameters satisfying $\beta+\gamma=\alpha+p-2$ and

$$
-1<\beta<p-2,-1<\gamma<p-2,
$$


then $f \in A_{\alpha}^{p}(D)$ if and only if

$$
\int_{D} \int_{D}\left(\frac{|f(z)-f(w)|}{|z-w|}\right)^{p} d A_{\beta}(z) d A_{\gamma}(w)<\infty .
$$

Remark 2. In [2], we proved one of the limit case of the above result, i.e., we proved that $f \in A_{-1}^{p}(D)(p>1)$ if and only if

$$
\int_{D} \int_{D}\left(\frac{|f(z)-f(w)|}{|z-w|}\right)^{p} d A_{\beta}(z) d A_{\gamma}(w)<\infty
$$

where $\beta=\gamma=(p-3) / 2$.

Taking $\beta=\gamma=\frac{p+\alpha-(n+1)}{2}$ in Theorems A and 1 we easily get the following corollary.

Corollary 2. Assume that $f \in H(B), \alpha>-1$ and $\alpha+n+1<p<\infty$. Then $f \in A_{\alpha}^{p}$ if and only if

(11) $\int_{B} \int_{B}\left(\frac{|f(z)-f(w)|}{\left|w-P_{w} z-s_{w} Q_{w} z\right|}\left(1-|z|^{2}\right)^{\frac{1}{2}}\left(1-|w|^{2}\right)^{\frac{1}{2}}\right)^{p} d v_{k}(z) d v_{k}(w)<\infty$ and if and only if

$$
\int_{B} \int_{B}\left(\frac{|f(z)-f(w)|}{|1-\langle z, w\rangle|}\left(1-|z|^{2}\right)^{\frac{1}{2}}\left(1-|w|^{2}\right)^{\frac{1}{2}}\right)^{p} d v_{k}(z) d v_{k}(w)<\infty
$$

where $k=(\alpha-(n+1)) / 2$.

Remark 3. An example in [9] shows that the formula (12) is no longer true in general when $p=n+1+\alpha$. When $0<p<n+1+\alpha$, in [3] we proved that $f \in A_{\alpha}^{p}$ if and only if

$$
\int_{B} \int_{B} \frac{|f(z)-f(w)|^{p}}{|1-\langle z, w\rangle|^{p}} d v_{\alpha}(z) d v_{\alpha}(w)<\infty .
$$

Note that the characterization for weighted Bergman space in Theorem A and Lemma 1 are different. Now, motivated by [1, 4], we find a new condition which interpolates these two conditions, i.e., we obtain the following theorem.

Theorem 2. Assume that $f \in H(B), 0 \leq q \leq p, \alpha>-1$ and $p>2 \alpha+2(n+1)$. Let $\beta=p / 2-(n+1)$ and $\gamma=\alpha+p / 2$. Then $f \in A_{\alpha}^{p}$ if and only if

$$
L:=\int_{B} \int_{B} \frac{|f(z)-f(w)|^{p-q}}{|1-\langle z, w\rangle|^{p}}|\widetilde{\nabla} f(z)|^{q} d v_{\beta}(z) d v_{\gamma}(w)<\infty .
$$

Proof. First of all $p>2 \alpha+2(n+1)$ implies $\beta>\alpha$. Now suppose that (13) holds. Making the change of variables $z \mapsto \varphi_{w}(z)$ and note that $\alpha=\gamma+\beta-p+n+1$ and $\beta=p / 2-(n+1)$, using the formula (6), we have

$$
L=\int_{B} \int_{B}|f(z)-f(w)|^{p-q}|\widetilde{\nabla} f(z)|^{q} \frac{\left(1-|z|^{2}\right)^{\beta+n+1}\left(1-|w|^{2}\right)^{\gamma}}{|1-\langle z, w\rangle|^{p}} d \lambda(z) d v(w)
$$




$$
\begin{aligned}
= & \int_{B} \int_{B}\left|f \circ \varphi_{w}(z)-f(w)\right|^{p-q}\left|\widetilde{\nabla} f \circ \varphi_{w}(z)\right|^{q}\left(1-|z|^{2}\right)^{\beta+n+1} \\
& \times\left(1-|w|^{2}\right)^{\beta+\gamma+n+1-p} d \lambda(z) d v(w) \\
= & \int_{B} \int_{B}\left|f \circ \varphi_{w}(z)-f(w)\right|^{p-q}\left|\widetilde{\nabla} f \circ \varphi_{w}(z)\right|^{q} d v_{\beta}(z) d v_{\alpha}(w) .
\end{aligned}
$$

Let $r>0$ and let $D(z, r)$ denote the Bergman metric ball at $z$ with radius $r$. By Lemma 2.4 of [11], there exists a positive constant $C$ such that

$$
|\nabla g(0)|^{p} \leq C \int_{D(0, r)}|g(z)-g(0)|^{p} d v_{\beta}(z)
$$

for all $g \in H(B)$. Replace $g$ by $f \circ \varphi_{w}$, we obtain

$$
\begin{aligned}
|\widetilde{\nabla} f(w)|^{p} & \leq C \int_{D(0, r)}\left|f \circ \varphi_{w}(z)-f(w)\right|^{p} d v_{\beta}(z) \\
& \leq C \int_{B}\left|f \circ \varphi_{w}(z)-f(w)\right|^{p} d v_{\beta}(z)
\end{aligned}
$$

Fix $w \in B$ for a moment and let $F_{w}(z)=f \circ \varphi_{w}(z)-f(w)$. Noting that $F_{w}(0)=0$, by Lemma 2 , we have

$$
\int_{B}\left|F_{w}(z)\right|^{p} d v_{\beta}(z) \leq C \int_{B}\left|F_{w}(z)\right|^{p-q}\left|\widetilde{\nabla} F_{w}(z)\right|^{q} d v_{\beta}(z) .
$$

In view of (14-16), we obtain

$$
\begin{aligned}
& \int_{B}|\widetilde{\nabla} f(w)|^{p} d v_{\alpha}(w) \\
\leq & C \int_{B} \int_{B}\left|F_{w}(z)\right|^{p} d v_{\beta}(z) d v_{\alpha}(w) \\
\leq & C \int_{B} \int_{B}\left|f \circ \varphi_{w}(z)-f(w)\right|^{p-q}\left|\widetilde{\nabla} f \circ \varphi_{w}(z)\right|^{q} d v_{\beta}(z) d v_{\alpha}(w) \\
= & C \int_{B} \int_{B} \frac{|f(z)-f(w)|^{p-q}}{|1-\langle z, w\rangle|^{p}}|\widetilde{\nabla} f(z)|^{q} d v_{\beta}(z) d v_{\gamma}(w) \\
= & C L<\infty .
\end{aligned}
$$

It follows from Lemma 1 that $f \in A_{\alpha}^{p}$.

Conversely, suppose that $f \in A_{\alpha}^{p}$. Since $\beta>\alpha$, we see that $f \in A_{\beta}^{p}$. By Lemma 2 and Theorem 2.16 of [11], it follows that

$$
\begin{aligned}
& \int_{B}\left|f \circ \varphi_{w}(z)-f(w)\right|^{p-q}\left|\widetilde{\nabla} f \circ \varphi_{w}(z)\right|^{q} d v_{\beta}(z) \\
\leq & C \int_{B}\left|f \circ \varphi_{w}(z)-f(w)\right|^{p} d v_{\beta}(z) \\
\leq & C \int_{B}\left|\widetilde{\nabla} f \circ \varphi_{w}(z)\right|^{p} d v_{\beta}(z)
\end{aligned}
$$




$$
=C \int_{B}|\widetilde{\nabla} f(z)|^{p}\left(1-\left|\varphi_{w}(z)\right|^{2}\right)^{\beta+n+1} d \lambda(z) .
$$

From (14) and (17) we obtain

$$
\begin{aligned}
L & =\int_{B} \int_{B}\left|f \circ \varphi_{w}(z)-f(w)\right|^{p-q}\left|\widetilde{\nabla} f \circ \varphi_{w}(z)\right|^{q} d v_{\beta}(z) d v_{\alpha}(w) \\
& \leq C \int_{B} \int_{B}|\widetilde{\nabla} f(z)|^{p}\left(1-\left|\varphi_{w}(z)\right|^{2}\right)^{\beta+n+1} d \lambda(z) d v_{\alpha}(w) .
\end{aligned}
$$

By using Fubini's Theorem, Lemma 3 and the condition $p>2 \alpha+2(n+1)$ we obtain

$$
\begin{aligned}
L & \leq C \int_{B}|\widetilde{\nabla} f(z)|^{p} d \lambda(z) \int_{B}\left(1-\left|\varphi_{w}(z)\right|^{2}\right)^{\beta+n+1}\left(1-|w|^{2}\right)^{\alpha} d v(w) \\
& =C \int_{B}|\widetilde{\nabla} f(z)|^{p} d v_{\beta}(z) \int_{B} \frac{\left(1-|w|^{2}\right)^{\alpha+\beta+n+1}}{|1-\langle z, w\rangle|^{2(\beta+n+1)}} d v(w) \\
& \leq C \int_{B}|\widetilde{\nabla} f(z)|^{p} d v_{\alpha}(z)<\infty,
\end{aligned}
$$

as desired.

Theorem 3. Suppose that $f \in H(B), 0<p<\infty$ and $\alpha>-1$. Then $f \in A_{\alpha}^{p}$ if and only if

$$
K:=\int_{B} \int_{B} \frac{|f(z)-f(w)|^{p}}{|1-\langle z, w\rangle|^{2 n+2}} d v_{\frac{n+1+\alpha}{2}}(z) d v_{\frac{n+1+\alpha}{2}}(w)<\infty .
$$

Proof. First we assume that (18) holds. For a fixed $r \in(0,1)$, let

$$
E(a, r)=\left\{z \in B:\left|\varphi_{a}(z)\right|<r\right\} .
$$

Set $|E(a, r)|=v(E(a, r))$. From [11] we see that

$$
\left(1-|z|^{2}\right)^{n+1} \asymp\left(1-|a|^{2}\right)^{n+1} \asymp|1-\langle a, z\rangle|^{n+1} \asymp|E(a, r)|
$$

when $z \in E(a, r)$. It is easy to see (using Cauchy's estimate for example) that there exists a positive constant $C$ such that

$$
|\nabla f(0)|^{p} \leq C \int_{E(0, r)}|f(z)-f(0)|^{p} d v(z)
$$

for all $f \in H(B)$. Replace $f$ by $f \circ \varphi_{w}$ and make a change of variables, we get

$$
|\widetilde{\nabla} f(w)|^{p} \leq C \int_{E(w, r)}|f(w)-f(z)|^{p} \frac{\left(1-|w|^{2}\right)^{n+1}}{|1-\langle z, w\rangle|^{2(n+1)}} d v(z)
$$

for all $f \in H(B)$ and $w \in B$. From the last inequality, (19) and Lemma 2.14 of [11], we have

$$
\begin{aligned}
& \left(1-|w|^{2}\right)^{p+\alpha}|\nabla f(w)|^{p} \\
\leq & \left(1-|w|^{2}\right)^{\alpha}|\widetilde{\nabla} f(w)|^{p}
\end{aligned}
$$




$$
\begin{aligned}
& \leq \frac{C}{\left(1-|w|^{2}\right)^{n+1-\alpha}} \int_{E(w, r)}|f(z)-f(w)|^{p} d v(z) \\
& \leq C \int_{E(w, r)} \frac{|f(z)-f(w)|^{p}}{|1-\langle z, w\rangle|^{2 n+2}}\left(1-|z|^{2}\right)^{\frac{n+1+\alpha}{2}}\left(1-|w|^{2}\right)^{\frac{n+1+\alpha}{2}} d v(z)
\end{aligned}
$$

Integrating both sides with respect to $d v$ on $B$, by (19) we get

$$
\begin{aligned}
& \int_{B}\left(1-|w|^{2}\right)^{p}|\nabla f(w)|^{p} d v_{\alpha} \\
= & \int_{B}\left(1-|w|^{2}\right)^{p+\alpha}|\nabla f(w)|^{p} d v \\
\leq & C \int_{B} \int_{E(w, r)} \frac{|f(z)-f(w)|^{p}}{|1-\langle z, w\rangle|^{2 n+2}}\left(1-|z|^{2}\right)^{\frac{n+1+\alpha}{2}}\left(1-|w|^{2}\right)^{\frac{n+1+\alpha}{2}} d v(z) d v(w) \\
\leq & C \int_{B} \int_{B} \frac{|f(z)-f(w)|^{p}}{|1-\langle z, w\rangle|^{2 n+2}} d v_{\frac{n+1+\alpha}{2}}(z) d v_{\frac{n+1+\alpha}{2}}(w) \\
= & C K<\infty .
\end{aligned}
$$

Hence $f \in A_{\alpha}^{p}$.

Conversely, suppose that $f \in A^{p}$. By Lemma 3 we obtain,

$$
\begin{aligned}
& K \leq C \int_{B} \int_{B} \frac{|f(z)|^{p}}{|1-\langle z, w\rangle|^{2(n+1)}}\left(1-|z|^{2}\right)^{\frac{n+1+\alpha}{2}}\left(1-|w|^{2}\right)^{\frac{n+1+\alpha}{2}} d v(z) d v(w) \\
& +C \int_{B} \int_{B} \frac{|f(w)|^{p}}{|1-\langle z, w\rangle|^{2(n+1)}}\left(1-|z|^{2}\right)^{\frac{n+1+\alpha}{2}}\left(1-|w|^{2}\right)^{\frac{n+1+\alpha}{2}} d v(z) d v(w) \\
& \leq C \int_{B}|f(z)|^{p}\left(1-|z|^{2}\right)^{\frac{n+1+\alpha}{2}} d v(z) \int_{B} \frac{\left(1-|w|^{2}\right)^{\frac{n+1+\alpha}{2}}}{|1-\langle z, w\rangle|^{2(n+1)}} d v(w) \\
& +C \int_{B}|f(w)|^{p}\left(1-|w|^{2}\right)^{\frac{n+1+\alpha}{2}} d v(w) \int_{B} \frac{\left(1-|z|^{2}\right)^{\frac{n+1+\alpha}{2}}}{|1-\langle z, w\rangle|^{2(n+1)}} d v(z) \\
& \leq C \int_{B}|f(z)|^{p} d v_{\alpha}(z)+C \int_{B}|f(w)|^{p} d v_{\alpha}(w) \\
& \leq C \int_{B}|f(z)|^{p} d v_{\alpha}(z)<\infty .
\end{aligned}
$$

The proof is complete.

Motivated by Theorem 3, similarly to Theorem 2 we obtain the following theorem.

Theorem 4. Assume that $f \in H(B), 0<p<\infty, 0 \leq q<p+2$ and $\alpha>-1$. Then $f \in A_{\alpha}^{p}$ if and only if

(20) $\quad M:=\int_{B} \int_{B} \frac{|f(z)-f(w)|^{p-q}}{|1-\langle z, w\rangle|^{2 n+2}}|\widetilde{\nabla} f(z)|^{q} d v(z) d v_{n+1+\alpha}(w)<\infty$. 
Proof. Suppose that (20) holds. By the proof of Theorem 2 we see that

$$
|\widetilde{\nabla} f(w)|^{p} \leq C \int_{B}\left|f \circ \varphi_{w}(z)-f(w)\right|^{p} d v(z) .
$$

After some computation, we get

$$
\begin{aligned}
M & =\int_{B} \int_{B}|f(z)-f(w)|^{p-q}|\widetilde{\nabla} f(z)|^{q} \frac{\left(1-|w|^{2}\right)^{n+1}\left(1-|z|^{2}\right)^{n+1}}{|1-\langle z, w\rangle|^{2(n+1)}} d \lambda(z) d v_{\alpha}(w) \\
& =\int_{B} \int_{B}\left|f \circ \varphi_{w}(z)-f(w)\right|^{p-q}\left|\widetilde{\nabla} f \circ \varphi_{w}(z)\right|^{q} d v(z) d v_{\alpha}(w) .
\end{aligned}
$$

Fix $w \in B$ at this moment, let $F_{w}(z)=f \circ \varphi_{w}(z)-f(w)$. Note that $F_{w}(0)=0$, by Lemma 2 we have

$$
\int_{B}\left|F_{w}(z)\right|^{p} d v(z) \leq C \int_{B}\left|F_{w}(z)\right|^{p-q}\left|\widetilde{\nabla} F_{w}(z)\right|^{q} d v(z)
$$

From (22) and (23), similarly to the proof of Theorem 2, we get

$$
\begin{aligned}
& \int_{B}|\widetilde{\nabla} f(w)|^{p} d v_{\alpha}(w) \\
\leq & C \int_{B} \int_{B}\left|f \circ \varphi_{w}(z)-f(w)\right|^{p-q}\left|\widetilde{\nabla} f \circ \varphi_{w}(z)\right|^{q} d v(z) d v_{\alpha}(w) \\
= & C \int_{B} \int_{B} \frac{|f(z)-f(w)|^{p-q}}{|1-\langle z, w\rangle|^{2 n+2}}|\widetilde{\nabla} f(z)|^{q} d v(z) d v_{n+1+\alpha}(w)=C M<\infty .
\end{aligned}
$$

Hence $f \in A_{\alpha}^{p}$.

Conversely, suppose that $f \in A_{\alpha}^{p}$. From (17) and (23) we obtain

$$
\begin{aligned}
M & =\int_{B} \int_{B}\left|f \circ \varphi_{w}(z)-f(w)\right|^{p-q}\left|\widetilde{\nabla} f \circ \varphi_{w}(z)\right|^{q} d v(z) d v_{\alpha}(w) \\
& \leq C \int_{B} \int_{B}|\widetilde{\nabla} f(z)|^{p} \frac{\left(1-|w|^{2}\right)^{n+1}}{|1-\langle z, w\rangle|^{2(n+1)}} d v(z) d v_{\alpha}(w) .
\end{aligned}
$$

Applying Fubini's Theorem, Lemmas 1 and 3 we have

$$
\begin{aligned}
M & \leq C \int_{B}|\widetilde{\nabla} f(z)|^{p} d v(z) \int_{B} \frac{\left(1-|w|^{2}\right)^{n+1}}{|1-\langle z, w\rangle|^{2(n+1)}} d v_{\alpha}(w) \\
& \leq C \int_{B}|\widetilde{\nabla} f(z)|^{p} d v_{\alpha}(z)<\infty
\end{aligned}
$$

The proof is complete.

Acknowledgments. The author of this paper is supported by the Educational Commission of Guangdong Province, China (No. LYM08092). 


\section{References}

[1] E. Kwon, A characterization of Bloch space and Besov space, J. Math. Anal. Appl. 324 (2006), no. 2, 1429-1437.

[2] S. Li, H. Wulan, R. Zhao, and K. Zhu, A characterisation of Bergman spaces on the unit ball of $\mathbb{C}^{n}$, Glasg. Math. J. 51 (2009), no. 2, 315-330.

[3] S. Li, H. Wulan, and K. Zhu, A characterization of Bergman spaces on the unit ball of $\mathbb{C}^{n}, I I$, Canad. Math. Bull. accepted.

[4] S. Li and S. Stević, Some characterizations of the Besov space and the $\alpha$-Bloch space, J. Math. Anal. Appl. 346 (2008), no. 1, 262-273.

[5] M. Nowak, Bloch space and Möbius invariant Besov spaces on the unit ball of $\mathbb{C}^{n}$, Complex Variables Theory Appl. 44 (2001), no. 1, 1-12.

[6] C. Ouyang, W. Yang, and R. Zhao, Characterizations of Bergman spaces and Bloch space in the unit ball of $\mathbb{C}^{n}$, Trans. Amer. Math. Soc. 347 (1995), no. 11, 4301-4313.

7] M. Pavlović and K. Zhu, New characterizations of Bergman spaces, Ann. Acad. Sci. Fenn. Math. 33 (2008), no. 1, 87-99.

[8] W. Rudin, Function Theory in the Unit Ball of $\mathbb{C}^{n}$, Springer-Verlag, New York-Berlin, 1980

[9] H. Wulan and K. Zhu, Lipschitz type characterizations for Bergman spaces, Canad. Math. Bull. 52 (2009), no. 4, 613-626.

[10] M. Zhang and W. Xu, Composition operators on $\alpha$-Bloch spaces of the unit ball, Acta Math. Sin. (Engl. Ser.) 23 (2007), no. 11, 1991-2002.

[11] K. Zhu, Spaces of Holomorphic Functions in the Unit Ball, Springer-Verlag, New York, 2005.

Department of Mathematics

JIAYING UNIVERSITY

514015, Meizhou, GuangDong, P. R. China

E-mail address: jyulsx@163.com 